\title{
Forgotten motives: The varieties of scientific experience ${ }^{*}$
}

\author{
by Yuri I. Manin ${ }^{\dagger}$
}

\author{
Le gros public: \\ A poêle, Descartes! à poêle!
}

(R. Queneau, Les Oeuvres complètes de Sally Mara)

When I arrived to Bures-sur-Yvette in May 1967, the famous seminar SGA 1966-67, dedicated to the Riemann-Roch theorem, was already drawing to an end. Mlle Rolland, then Léon Motchane's secretary at the IHÉS, found for me a nice small apartment in Orsay. Each early morning, awoke to the loud chorus of singing birds, I walked to Bures, anticipating the new session of private tutoring on the then brand-new project of motives, by the Grand Maître himself, Alexander Grothendieck. Several pages, written by his hand then, survived in my archive, in particular, the one dedicated to the "Standard Conjectures". These conjectures remain unproved after half of century of vain efforts. Grothendieck himself saw them as the cornerstone of the whole project. In his letter to me dated March 20, 1969, he has written:

Je dois avouer à ma honte que je ne sais plus distinguer à première vue ce qui est démontrable (voire plus ou moins trivial) sans les conjectures standard, et ce qui ne l'est pas. C'est évidemment honteux qu'on n'ait pas démontrées les conjectures standard!

Still, during the decades that passed since then the vast realm of motives kept rewarding humility of many researchers prepared to be happy with what they could do using the tools they could elaborate.

Several times Grothendieck invited me to his house at rue de Moulon. He allowed me to browse

* Copyright (c) 2014 Yuri I. Manin. Used with permission. This article appears also in the book Alexandre Grothendieck: A Mathematical Portrait, edited by Leila Schneps, International Press, June 2014.

† Max-Planck-Institut für Mathematik, Bonn, Germany

E-mail:manin@mpim-bonn.mpg.de through his bookshelves; I borrowed a few books to read at home. When I last visited him a day or two before my departure, I asked him to sign for me his book or paper. To my amazement, he opened "Les CEuvres complètes de Sally Mara” by Raymond Queneau and scribbled at the first page:

\section{Hommage affectueux R. Queneau}

\section{Early History of Motives}

Having returned to Moscow in June 1967, after five or six weeks of intense training with Grothendieck, I spent several months writing down his main definitions related to motives and studying necessary background material in the literature. I was very pleased when it turned out that I could answer one of his questions and calculate the motive of a blow-up without using standard conjectures. My paper [Ma68] containing this exercise was submitted next summer and published in Russian. It became the first ever publication on motives, and Grothendieck recommended it to David Mumford (in his letter of April 14, 1969) as "a nice foundational paper" on motives.

To me Grothendieck has written in Russian the letter about this paper (05/02/1969). This seems to be the only document showing that he had some Russian, probably, learned from his father.

The first step in the definition of a category of (pure) motives is this. We keep objects of a given algebraic-geometric category, say of smooth projective varieties over a fixed field $\operatorname{Var}_{k}$, but replace its morphisms by correspondences. This passage implies that morphisms $X \rightarrow Y$ now form an additive group, or even a $K$-module rather than simply a set, where 
$K$ is an appropriate coefficient ring. Moreover, correspondences themselves are not just cycles on $X \times$ $Y$ but classes of such cycles modulo an "adequate" equivalence relation. The coarsest such relation is that of numerical equivalence, when two equidimensional cycles are equivalent if their intersection indices with each cycle of complementary dimension coincide. The finest one is the rational (Chow) equivalence, when equivalent cycles are deformations over a base which is a chain of rational curves. Direct product of varieties induces tensor product structure on the category.

The second step in the definition of the relevant category of pure motives consists in a formal construction of new objects (and relevant morphisms) that are "pieces" of varieties: kernels and images of projectors, i.e. correspondences $p: X \rightarrow$ $X$ with $p^{2}=p$. This produces a pseudo-abelian, or Karoubian completion of the category. In this new category, the projective line $\mathbf{P}^{1}$ becomes the direct sum of the (motive of) a point and the Lefschetz motive $\mathbf{L}$ (intuitively corresponding to the affine line).

The third, and last step of the construction, is one more formal enhancement of the class of objects: they now include all integer tensor powers $\mathbf{L}^{\otimes n}$, not just non-negative ones, and tensor products of them with other motives.

Various strands of intuition are interwoven in this fundamental pattern discovered by Grothendieck, and I will now try to make them (more) explicit.

The basic intuition that guided Grothendieck himself, was the image of the category of pure Chow motives $\mathrm{Mot}_{k}$ as the receptacle of the "universal cohomology theory" $V_{k} \rightarrow$ Mot $_{k}: V \mapsto h(V)$. The universal theory was needed in order to unite various cohomological constructions, such as Betti, de Rham-Hodge, and étale cohomology.

What looked paradoxical in this image was the following observation about transcendental cycles on an algebraic variety $X$. One could get hold of these cycles for $k=\mathbf{C}$ by appealing to algebraic topology, or else, to complicated constructions of homological algebra involving all finite covers of $X$.

But in the category of pure motives, one from the start dealt only with algebraic cycles, represented by correspondences, and it was intuitively not at all clear how on earth they could convey information about transcendental cycles. Indeed, the main function of the "Standard Conjectures" was to serve as a convenient bridge from algebraic to transcendental. Everything that one could prove without them was indeed "plus ou moins trivial" - until people started treating correspondences themselves using sophisticated homological algebra (partly generated by the development of étale cohomology and Grothendieck-Verdier introduction of derived and triangulated categories).
However, the passage from the set of morphisms to the $K$-module of correspondences involves one more intuitive idea, and it can be most succinctly invoked by referring to physics, namely the great leap from the classical mode of description of nature to the quantum one. This leap defined the science of the XXth century. Its basic and universal step consists in the introduction of a linear span of everything that in classical physics was only a set: points of a phase space, field configurations over a domain of space-time etc. Such quantum superpositions then form linear spaces on which Hilbert-like scalar products are defined, that in turn allow one to speak about probability amplitudes, quantum observations etc.

I have no evidence that Grothendieck himself thought then about quantum physics in relation to his algebraic geometry project. We do know that concerns about weapons of mass destruction and collaborationist behaviour of scientists towards their governments and military-industrial complexes inspired in him deep disturbance and aversion. The most direct source of his inspiration might have been algebraic topology that after 1940's put more stress upon chains and cochains than upon simplices and the ways they are glued together.

However, in my personal development as a mathematician in the 1970's-80's and later, the study of quantum field theory played a great role, and feedback from theoretical physics to algebraic geometry, that was ahead, became for me a great source of inspiration. I was and remain to be possessed by a Cartesian dream, poetic rationalism, whatever history has yet to say about Der Untergang des Abendlandes.

Below I will sketch a map of two branches of the development of Grothendieck ideas about motives that approximately followed two intuitions invoked above: from homological algebra and from physics respectively. The references at the end of this essay constitute the bare minimum of the relevant research, but the reader will be able to find much additional material bibliography in the survey collection [Mo91] and in [A04], [VoSuFr00], [Ta11].

\section{Motives and Homological Algebra}

The most common linear objects are modules over rings in algebra and sheaves of modules in algebraic geometry. Free modules/locally free sheaves are closest to classical linear spaces.

General algebraic variety $X$, or a scheme, is a highly non-linear object.

In the classical algebraic geometry over, say, complex numbers, the variety $X$ used to be identified with the topological space $X(\mathbf{C})$ of its $\mathbf{C}$-points, and could be studied by topological methods involving triangulations or cell decompositions. In the geometry over, 
say, finite fields, this did not work, and when André Weil in 1949 stated his famous suggestion that point count over finite fields should be done using trace of the Frobenius endomorphism acting upon appropriately defined (co)homology groups of $X$, this generated a flow of research.

The first product of this research was the creation of the cohomology theory of coherent sheaves of modules $\mathcal{F}$ on varieties $X$ or more general schemes. Now, in a constructive definition of $H^{*}(X, \mathcal{F})$, one could either stress combinatorics of covers of $X$ by open sets in the Zariski topology (Čech cohomology) or, alternatively, "projective/injective resolutions" of $\mathcal{F}$, that is special exact complexes of sheaves $\cdots \rightarrow$ $F_{2} \rightarrow F_{1} \rightarrow F_{0}:=\mathcal{F} \rightarrow 0$ or similarly with arrows inverted. This passage from the dependence of $H^{*}(X, \mathcal{F})$ on the non-linear argument $X$ to the dependence on the linear argument $\mathcal{F}$ was very characteristic for the early algebraic geometry of 1950's and 1960's. "Homological Algebra" by H. Cartan and S. Eilenberg, the famous FAC, "Faisceaux Algébriquets Cohérents" by J.-P. Serre, became the standard handbooks for every aspiring young algebraic geometer.

David Mumford and I started our training as algebraic geometers at the same time, about 1956, he at Harvard, I at Moscow University. David reminisces that his teacher Zariski "was motivated by the need to make the work of the Italian school rigorous by using the new methods of commutative algebra". My teacher Shafarevich suggested us as well to study glorious Italian algebraic geometry, approaching it armed with modern insights and techniques developed by Serre, Grothendieck and their school.

I had no time nor use for a course of "Instant Italian", so I've been reading two books simultaneously, "Le Superficie Algebriche" by Federigo Enriques (Zanichelli, 1949) and "Divina Commedia", and each time that I opened Enriques (or for that matter, SGA), I recited mournfully: ... lasciate ogni speranza voi ch'entrate...

Nevertheless, it worked. When I brought from $\mathrm{Bu}$ res in 1967 xeroxed papers by Gino Fano, Vassya Iskovskikh and I could read them without bothering much in which language they have been written, and then produce the first examples of birationally rigid varieties, and unirational but not rational threefolds using Fano methods.

Homological algebra proved more resistant, and here I learned most of what I understand now from the next generation of eager young Moscow students, by now long ago mature researchers themselves.

We first learned, of course, about the basic Grothendieck-Verdier presentation of homological algebra as the theory of derived, and more generally, triangulated categories. Passage from the Bourbaki language of structures to the now domineering language of categories (and then polycategories) involved several radical changes of intuition, and as it is now clear, led in the garden of forking paths. The passage from one crossroad to another one always involved a decision about what should be disregarded, and later it could happen - and always happened that one was bound to turn back again and to recollect some forgotten ideas.

The story of derived categories started with categories, whose objects were complexes (of abelian groups/sheaves/objects of an abelian category) considered modulo homotopy.

On the stage of Grothendieck-Verdier triangulated categories, one forgot about initial objects-complexes and focused on an abstract additive category, endowed with a translation functor and a class of diagrams, called distinguished triangles. But the problem of nonfunctoriality of cones led back to the complexes of abelian groups, this time upgraded to the level of morphisms rather than objects.

This was, of course, a special case of enriched categories, which in the simplest incarnation postulate Bourbaki-structured morphism sets $\operatorname{Hom}(X, Y)$, but with an upgrading: this time one clearly had to deal with the case of categorified morphism sets. However, when one allows morphisms to be objects of a category, then morphisms of this second floor category might form a category as well ... and we find ourselves ascending the Tower of Babel that could cause despair even in Grothendieck himself.

For the limited purposes of this note, I will disregard subtleties and various versions of the notion of triangulated/dg-categories, and will only sketch several basic discoveries of the last decades relating such categories with motives.

Roughly speaking, starting with a category of varieties (or schemes) $X$, one may consider either the replacement of each $X$ by a triangulated category $D(X)$ of complexes of (quasi)-coherent sheaves on $X$, or else return to the initial Grothendieck insight, but replace correspondences by complexes of correspondences.

The latter approach led to the Voevodsky's motives ([VoSuFr00]). I will focus on some achievements of the first one.

One of the first great surprises was Alexander Beilinson's discovery ([Be83]) that a derived category of a projective space can be described as a triangulated category made out of modules over a Grassmann algebra. In particular, a projective space became "affine" in some kind of non-commutative geometry! The development of Beilinson's technique led to a general machinery describing triangulated categories in terms of exceptional systems and extending the realm of candidates to the role of non-commutative motives. 
D. Orlov ([Or05]) proved a general theorem to the effect that if $X, Y$ are smooth projective $k$-varieties and if there is a fully faithful functor $F: D^{b}(X) \rightarrow$ $D^{b}(Y)$, then the Chow motive $h(X)$ is a direct summand of $h(Y)$ "up to translations and twists by Lefschetz/Tate motives".

M. Kontsevich formalised the properties of $d g$-categories, expressing properness and smoothness in case of the derived categories of varieties, and defined the respective class of categories (modulo homotopy) as "spaces" in non-commutative algebraic geometry. He then defined the respective class of Chow motives and has shown that there exists a natural fully faithful functor embedding Grothendieck's Chow motives (modulo twists) into non-commutative motives. These ideas were further developed by Tabuada, Marcolli, Cisinski et al., cf. the recent survey [Ta11] and references therein.

\section{Motives and Physics}

At mid-1970's and further on, algebraic geometry interacted with physics more intensely that ever before: self-dual gauge fields (instantons), completely integrable systems (Korteweg-de Vries equations), emergence of supergeometry (based upon formal rules of Fermi statistics), the Mumford form and the Polyakov measure on moduli spaces of curves (quantum strings) have been discussed at joint seminars and local and international conferences of physicists and mathematicians.

Motives did not yet appear in this picture. However, in 1991 something new and unexpected happened.

B. Greene in his book "The Elegant Universe. Superstrings, Hidden Dimensions and the Quest for the Ultimate Theory" tells the following story:

"At a meeting of physicists and mathematicians in Berkeley in 1991, Candelas announced the result reached by his group using string theory and mirror symmetry: 317206 375. Ellingsrood and Strømme announced the result of their very difficult mathematical computation: 2682549 425. For days, mathematicians and physicists debated: Who was right? [...]

About a month later, an e-mail message was widely circulated among participants in the Berkeley meeting with the subject heading: Physics Wins! Ellingsrood and Strømme had found an error in their computer code that, when corrected, confirmed Candelas's result."

The problem about which Greene speaks is this. Consider a smooth hypersurface $V$ of degree 5 in $\mathbf{P}^{4}$. Denote by $n(d)$ the (appropriately defined) number of rational curves of degree $d$ on $V$. Calculating $n(d)$ looks like perfectly classical problem of enumerative algebra geometry, and in fact the numbers $n(1)=2875$ and $n(2)=609250$ were long known. The physicists Ph. Candelas, X. C. de la Ossa, P. S. Green, and L. Parkes using machinery and heuristics of quantum string theory, calculated not just $n(3)$, they have given an analytic expression for a total generating function for these numbers, using the so called Mirror Conjecture. The mathematicians G. Ellingsrood and S. A. Strømme produced a computer code calculating $n(3)$.

Omitting a lot of exciting developments of this rich story, I will briefly explain only the part that refers to the new and highly universal motivic structure that emerged in algebraic geometry. I will speak about varieties, although in fact Deligne-Mumford stacks form the minimal habitat for this structure, and the respective extension of the construction of pure motives for them is needed; this was done by B. Toën.

Roughly speaking, we now treat the general problem, inherited from classical enumerative geometry: given a projective variety $V$, (define and) calculate the number of algebraic curves of genus $g$ on $V$, satisfying additional incidence conditions that make this number finite, as in Euclidean archetype: "one line passes through two different points of plane". After considerable efforts, one can define for all stable values of $(g, n)$ a Chow class $I_{g, n}$ on $V^{n} \times \bar{M}_{g, n}$ with coefficients in the completed semi-group ring, say $\mathbf{Q}\left[\left[q^{\beta}\right]\right]$ where $\beta$ runs over integral classes in the Mori cone of $V$. This class expresses the virtual incidence relation, described above, by reducing it to the positions of the respective points in $V^{n}$ on the one hand, and to the position of the respective curve in the Deligne-Mumford stack of curves of genus $g$ with $n$ marked points.

When this is done, a list of universal properties of the classes $I_{g, n}$ treated as motivic morphisms, defines essentially the (co)action of the modular (co)operad with components $h\left(\bar{M}_{g, n}\right)$ in the category of motives upon each total motive $h(V)$ (I use the word total in order to stress that we are not allowed to pass to pieces here, although twisting and translations are in fact present, cf. [BehM96]).

Sophistication of both theoretical (and imaginative) physics and abstract mathematics that cooperated to discover this picture is really amazing, and I would like to draw attention to the fact that our traditional (mis)representation of mathematics as a language and technical tool needed to make physical intuition precise, was reversed here: physical intuition helped discover mathematical structures that were not known before. One remarkable result of this was Deligne's generalisation of the Tannakian Galois formalism ([De02]): it turned out that motivic Galois groups are actually supergroups, so that the Fermi statistics now firmly resides in algebraic geometry as well, which up to then was "purely bosonian".

Of course, such reversal had also happened many times in history, but here the contemporary status 
of both theory of motives and quantum strings adds a strong romantic touch to the story. The beautiful two-volume cooperative project of the two communities trying to enlighten each other, [QFS99], is branded by two epigraphs. Epigraph to the first volume is a quotation from Grothendieck's "Récoltes et Semailles":

Passer de la mécanique quantique de Newton à celle d'Einstein doit être un peu, pour le mathématicien, comme de passer du bon vieux dialecte provençal à l'argot parisien dernier cri. Par contre, passer à la mécanique quantique, j'imagine, c'est passer du français au chinois.

(In the pre-post-modern times one would have said: "It's all Greek to me!").

The second volume starts with epigraph, written in Chinese logograms, from Confucius' "Analects", 17:2. Here I give its translation:

The Master said: "Men are close to one another by nature. They drift apart through behaviour that is constantly repeated".

This is the collective riposte of the two communities, arguing their closeness, but in the language that is foreign for both.

$$
* * *
$$

In his letter to me from Les Aumettes dated March 8,1988 , the last letter that I have, Grothendieck has written:

... thanks for your letter of birthday congratulations, and please excuse my being late in replying to this letter, as well as the previous one and thanking for the reprint with dedication of november last year. Your letter struck me as somewhat formal and kind of ill at ease, and surely my silence has contributed to it. What I had to say about the spirits in mathematics today I said in the volumes I sent you and a number of other former friends. I am confident that before the year 2000 is reached, mathematicians (and even non-mathematicians) will read it with care and be amazed about times strange at last left behind ...

I met Grothendieck almost half a century ago. Thinking back on his imprint on me then, I realise that it was his generosity and his uncanny sense of humour that struck me most, the carnivalistic streak in his nature, which I later learned to discern in other anarchists and revolutionaries.

On the front cover of the issue no 14 of "Survivre ... et Vivre" (Octobre-novembre 1972) that miraculously reached me by post in Moscow, I read:

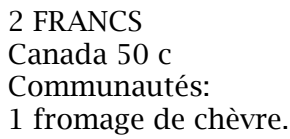

\section{References}

[Gr69] A. Grothendieck. Standard conjectures on algebraic cycles. In: Algebraic Geometry, Bombay Colloquium, 1968, Oxford UP, 1969, pp. 193-199.

[Ma68] Yu. Manin. Correspondences, motives and monoidal transformations. Math. USSR-Sb., 6 (1968), pp. 439-470.

[Ja92] U. Janssen. Motives, numerical equivalence, and semi-simplicity. Invent. Math. 107 (1992), 447-452.

[Mo91] Motives. Proc. Summer Research Conference, 1991. Eds. U. Janssen, S. Kleiman, J.-P. Serre. Proc. Symp. in Pure Math., vols. 55.1, 55.2, AMS 1994.

[VoSuFr00] V. Voevodsky, A. Suslin, E. Friedlander. Cycles, transfers, and motivic homology theories. Ann. Math. studies, Princeton UP, 2000.

[A04] Y. André. Une Introduction aux Motifs (motifs purs, motifs mixtes, périodes). Panoramas et Synthéses, Nr. 17, Soc. Math. de France, 2004.

[Or05] D. Orlov. Derived categories of coherent sheaves and motives. arXiv:math/0512620.

[Be83] A. Beilinson. The derived category of coherent sheaves on $\mathbf{P}^{n}$. Selecta Math. Soviet. 3, 1983, pp. 233-237.

[Ta11] G. Tabuada. A guided Tour through the Garden of Noncommutative Motives. arXiv:1108.3787.

[QFS99] Quantum Fields and Strings: A Course for Mathematicians. Vols. 1, 2. Ed. by P. Deligne, Ed Witten, et al., AMS, 1999.

[De02] P. Deligne. Catégories tensorielles. (Dedicated to Yuri I. Manin on the occasion of his 65th birthday.) Mosc. Math. J. 2 (2002), no. 2, 227-248.

[BehM96] K. Behrend, Yu. Manin. Stacks of stable maps and Gromov-Witten invariants. Duke Math. J., 85, no.1, 1996, pp. 1-60. 